\title{
An Initial Study \\ of the \\ Fundamentals of Ice Crystal Icing Physics \\ in the NASA Propulsion Systems Laboratory
}

\author{
June 5-9, 2017 \\ AIAA Aviation Conference
}

Peter Struk

NASA Glenn Research Center

Tadas Bartkus, Jen-Ching Tsao

Ohio Aerospace Institute

Timothy Bencic, Michael King, Thomas Ratvasky, Judy Van Zante

NASA Glenn Research Center 


\section{Outline}

- Introduction \& background

- NASA Fundamental Ice Crystal Icing Research Goals

- Concepts using the NASA Propulsion System Laboratory (PSL)

- Experimental Description

- Results

- Freeze-out characteristics of cloud

- Changes in aero-thermal conditions at the test section

- Accreted ice characteristics observed

- Summary 


\section{Introduction}

Advance Air Transport Technology

Project (AATT; 2015 +)

Advanced Aircraft Icing (AAI) Subproject

- Challenging to study ice-accretion physics directly inside the engine

- Trying to simulate local ICI environment without using an engine

- This paper presents an initial study of the fundamental physics of ICI using PSL

- Test occurred in March 2016

- Select results presented

- Last year, presented preliminary work in preparation for this test

- Complementary papers to follow

\section{Technical Challenge:}

Expand engine aero-thermodynamic modeling capability to predictively assess the onset of icing in current and $\mathrm{N}+2 / \mathrm{N}+3$ aircraft during flight operation (FY21).

The simulation tools are well anchored in results from both fundamental physics studies and full engine tests. 


\section{NASA Fundamental ICI Research Goals}

- Identify and bound the conditions affecting icecrystal ice accretion at the (local) accretion site

- Generate \& characterize (i.e. measure) those conditions

- Gather data and develop models on ice-crystal icing factors
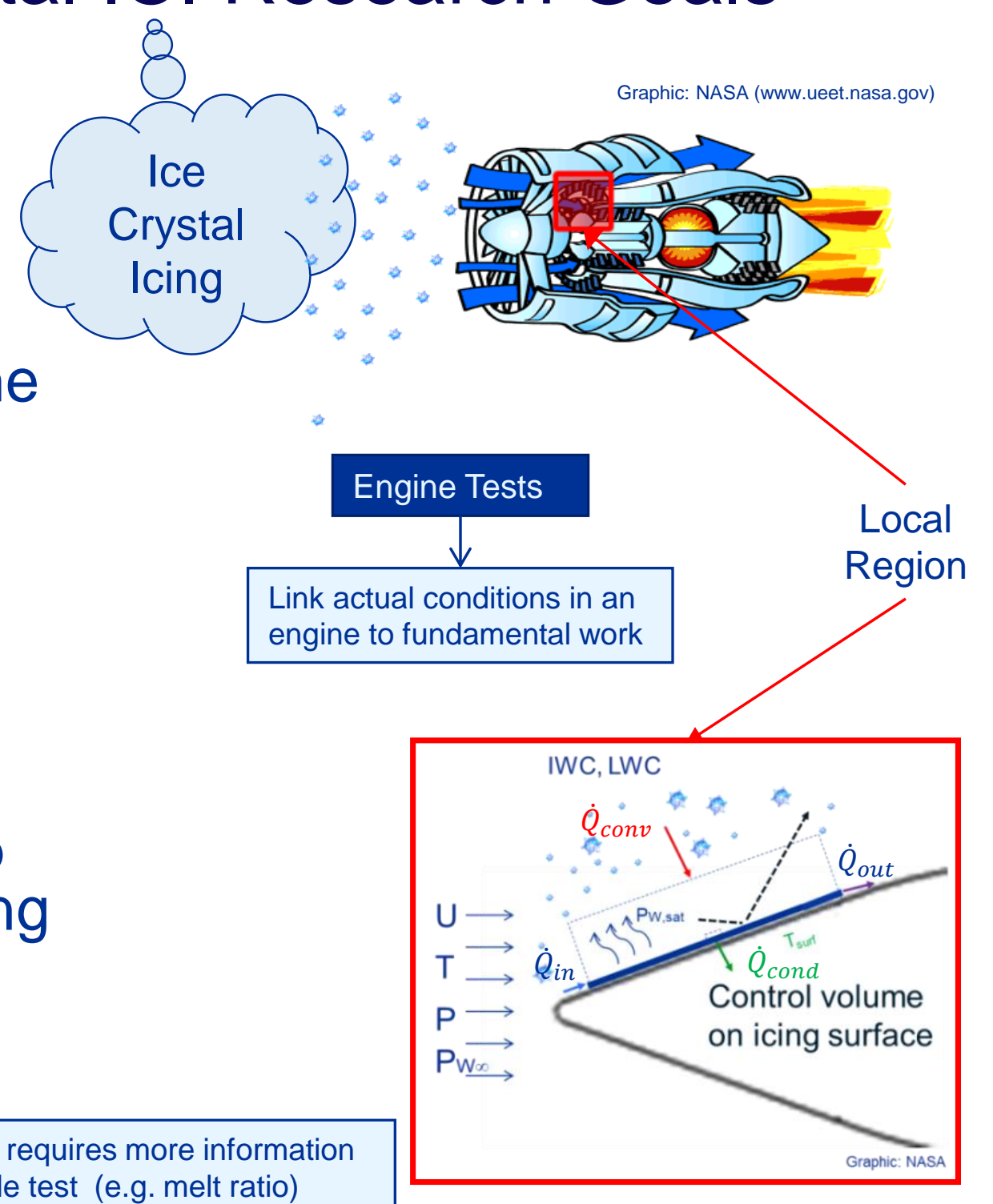


\section{Concept Using PSL}

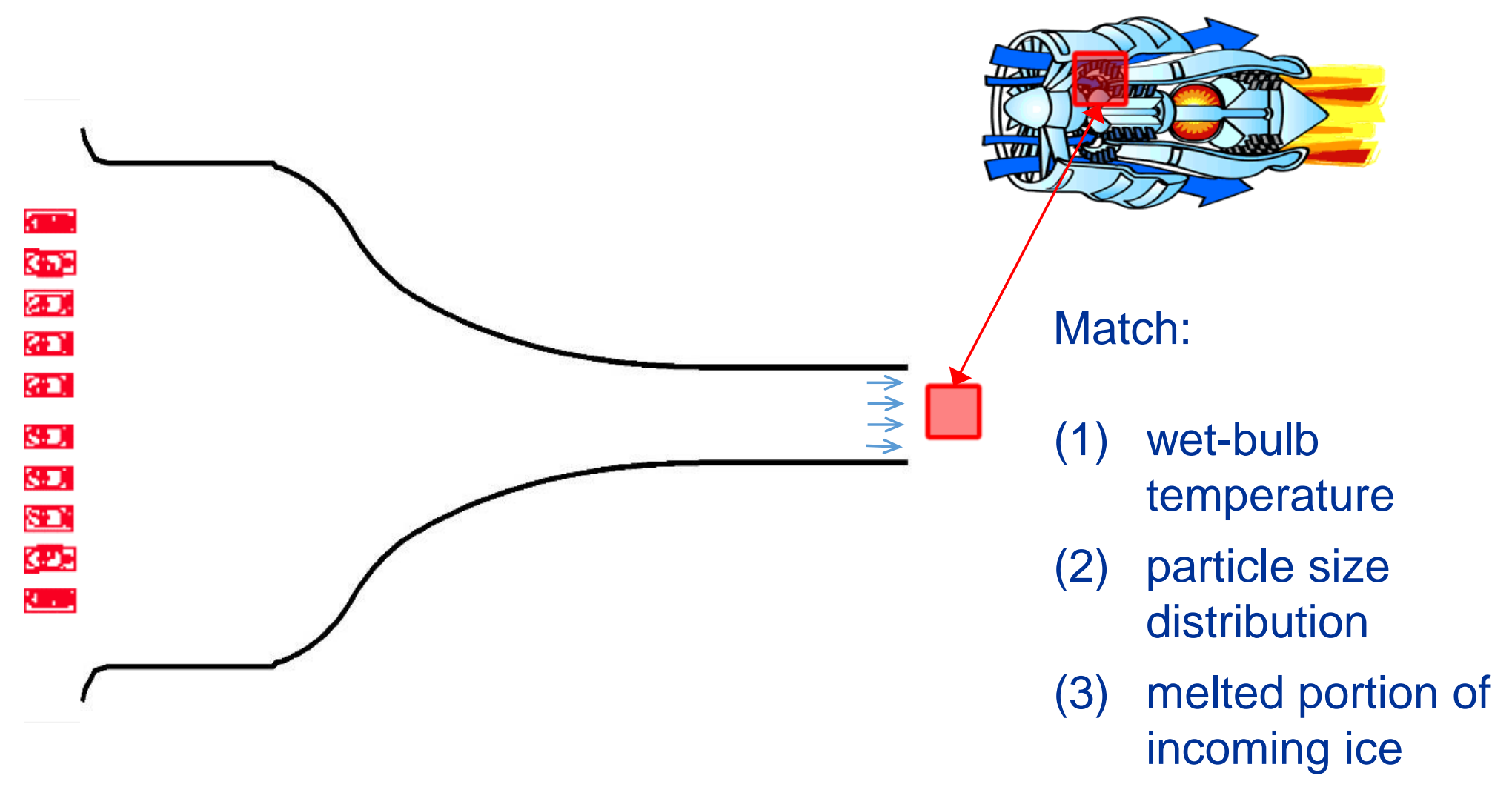

Goal: Ability to generate a prescribed mixed-phase condition at the test section for fundamental ice-crystal icing research 


\section{Fundamental Test \#1}

- Eight (8) days of testing occurred in March 2016

- Objectives

- Examine spray bar and plenum parameters and how they affect the mixedphase at the exit of the free jet

- Cloud characterization at the test section:

- Melt ratio (fraction of freeze out)

- Total water content

- Temperature \& humidity measurements at test section (cloud on vs. cloud off)

- Particle size distributions

- Uniformity

- Observe ice accretion 


\section{PSL Configuration}

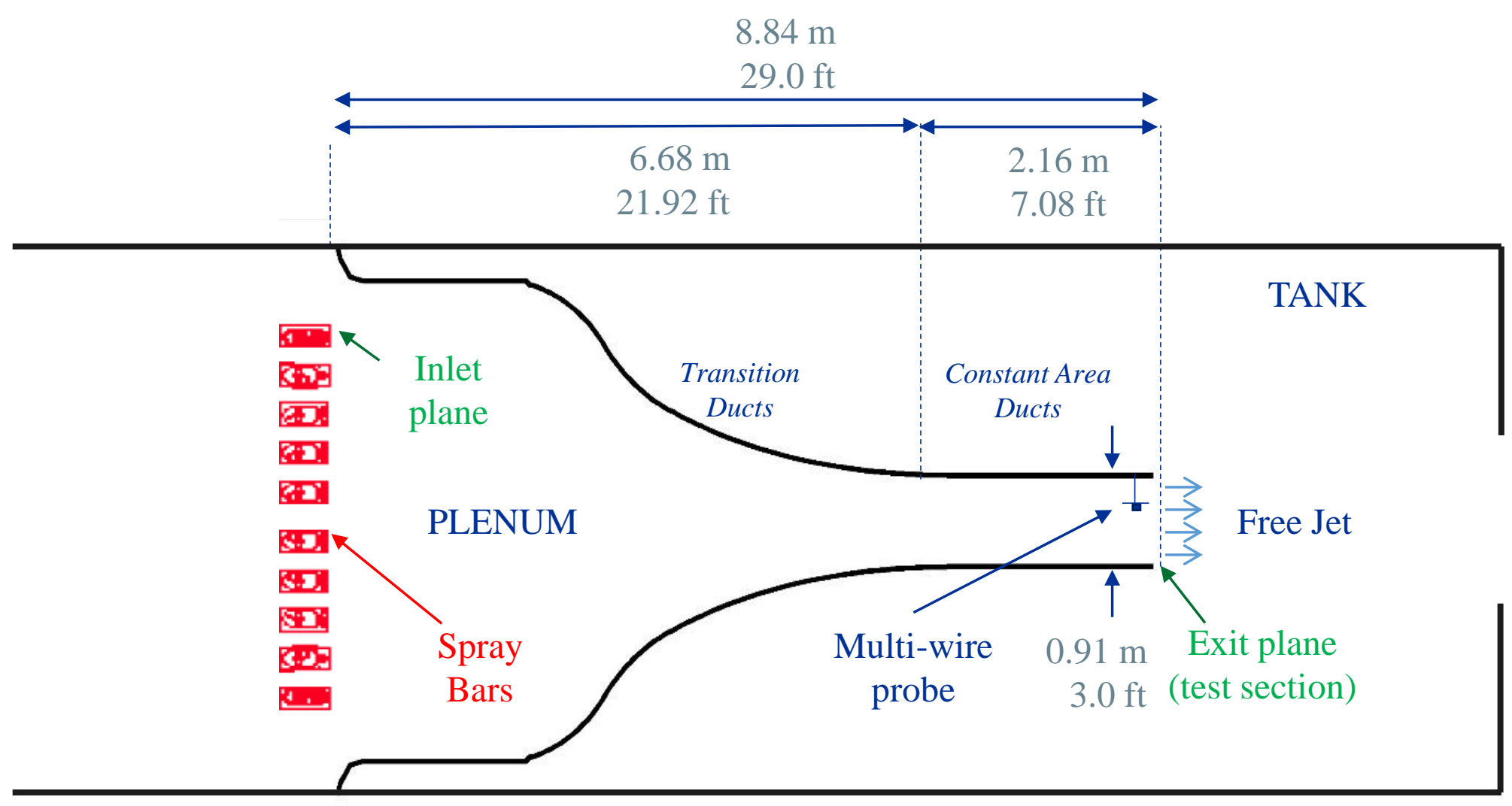




\section{Test Configurations}
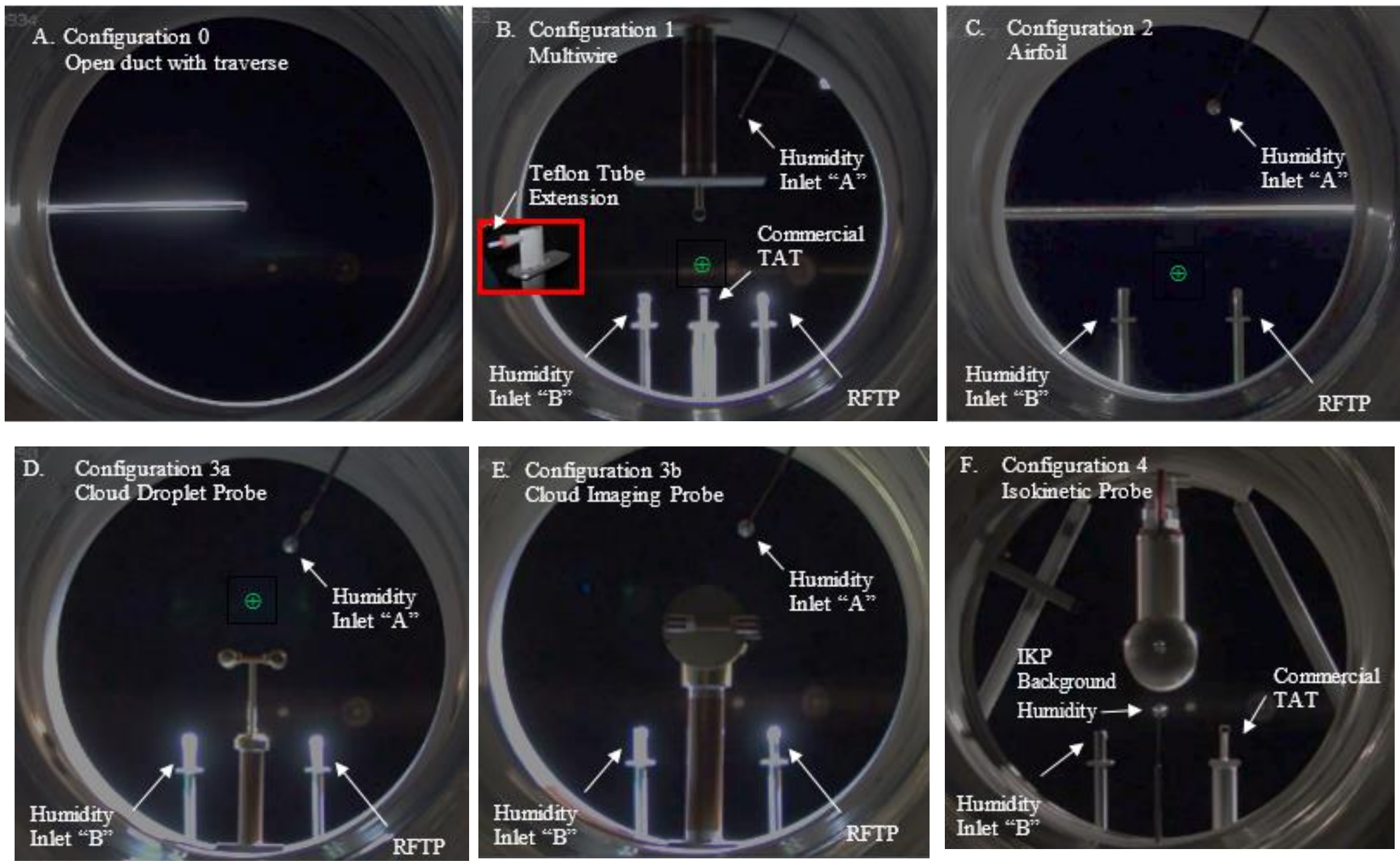


\section{Mixed-Phase Investigation \\ Plenum Relative Humidity Sweep Approach}

\section{Parameters}

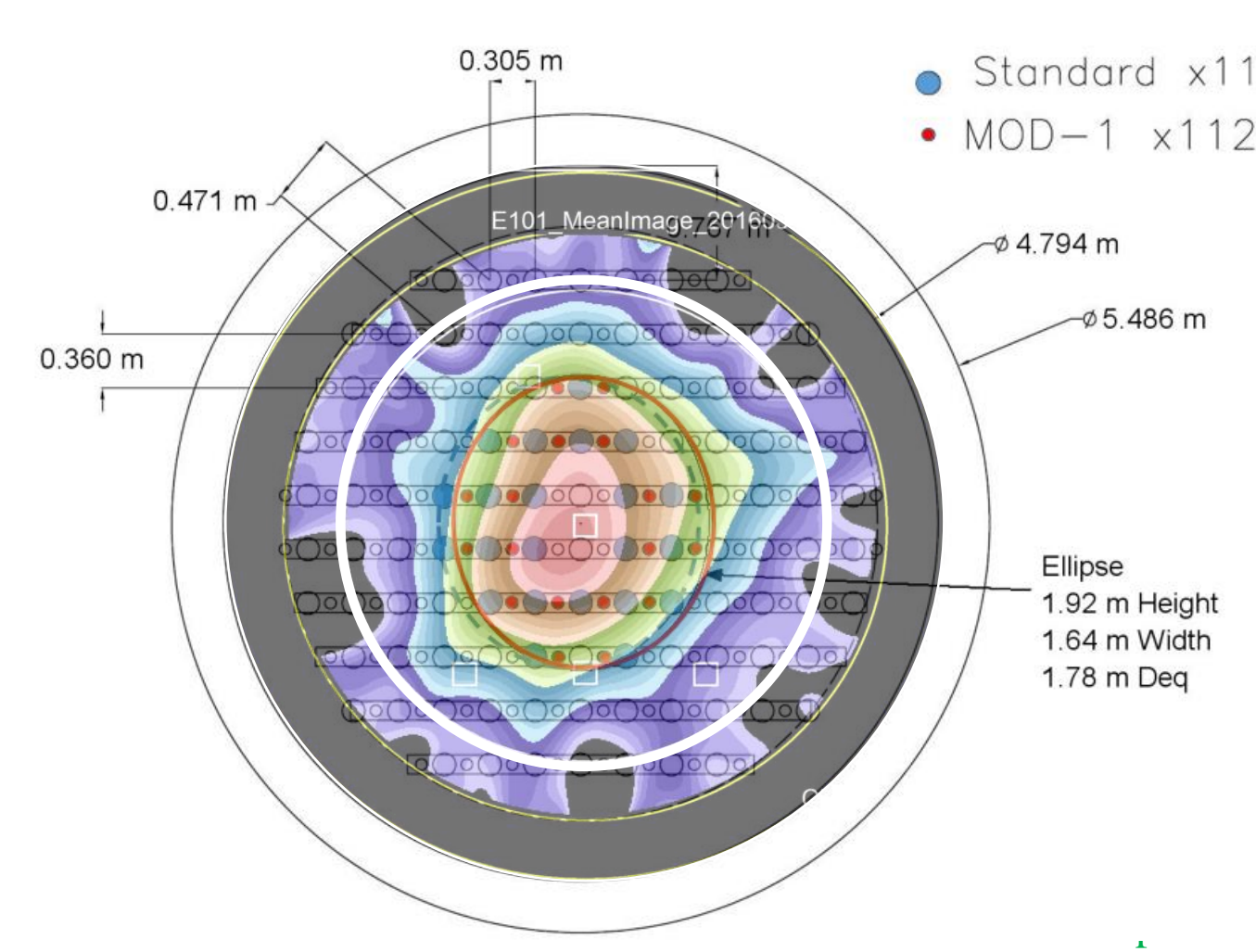

Nomenclature

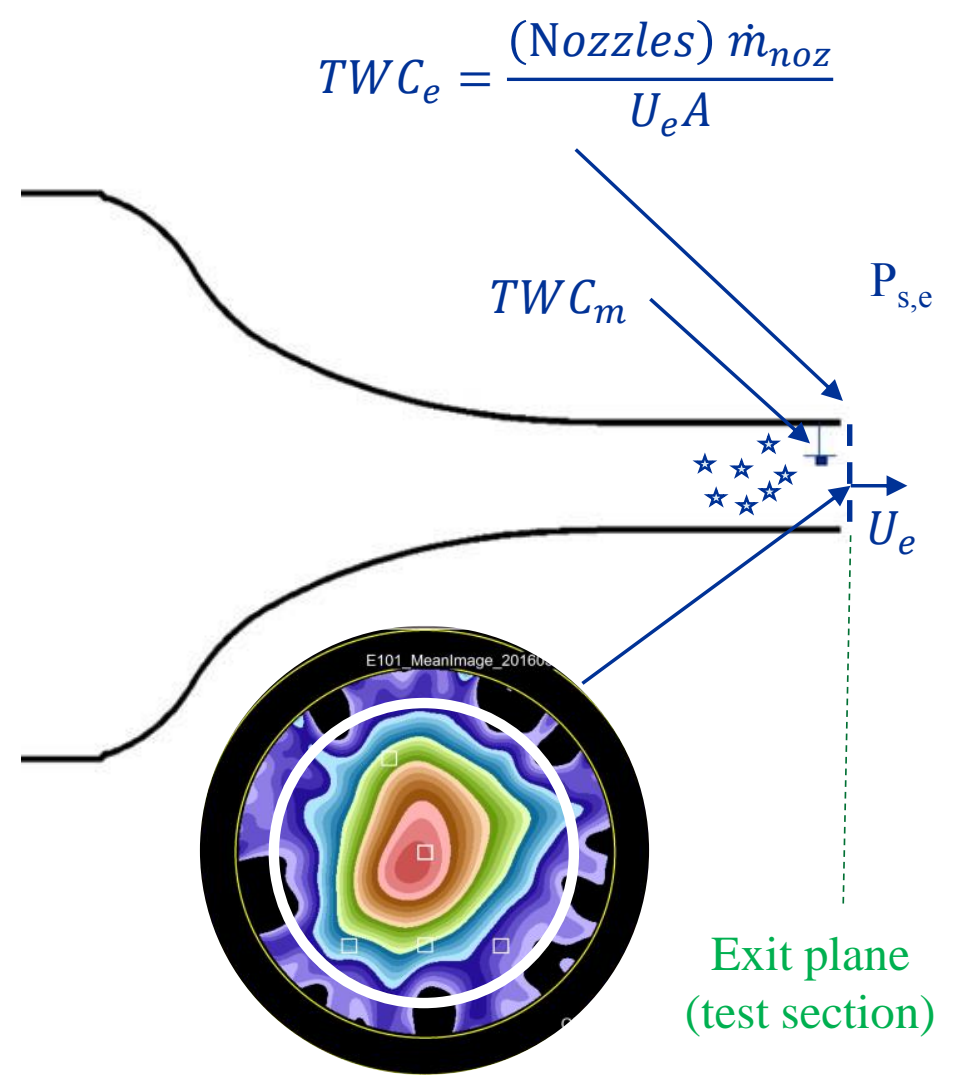




\section{Mixed-Phase Investigation}

\section{Plenum Relative Humidity Sweep Results}

\section{Water Content Measurement Results}

$$
\begin{aligned}
& \mathrm{P}_{0, \mathrm{i}, \mathrm{T}}=44.8 \mathrm{kPa}(6.5 \mathrm{psia}) \text { and } 42.8 \mathrm{kPa}(6.21 \mathrm{psia}) \\
& \mathrm{T}_{0, \mathrm{i}, \mathrm{T}}=7.2^{\circ} \mathrm{C} \\
& \left.\mathrm{TWC}_{\mathrm{e}, \mathrm{T}}=6.5 \mathrm{~g} / \mathrm{m}^{3} \text { ( }^{*} \text { Estimated }\right)
\end{aligned}
$$
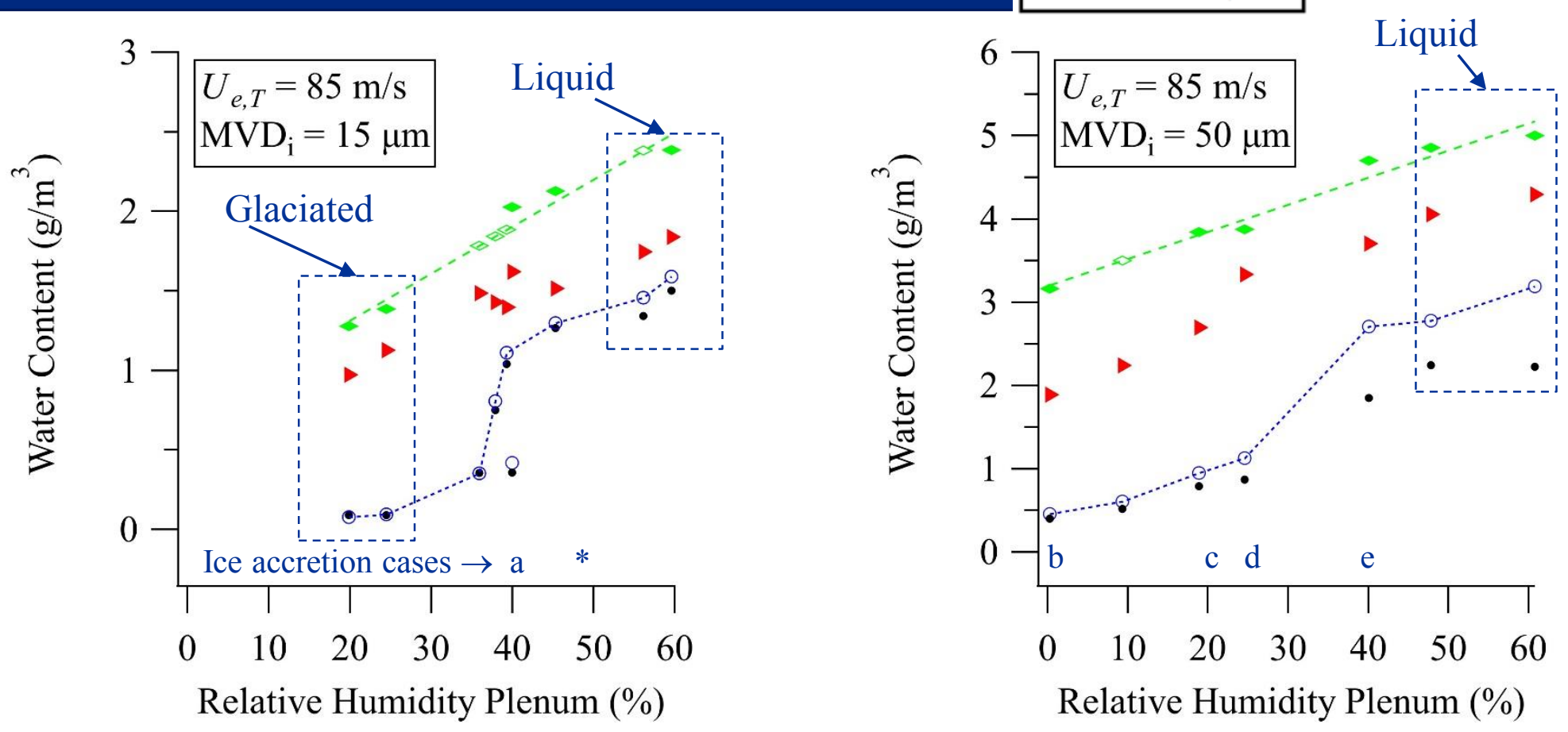


\section{Mixed-Phase Investigation}

\section{Plenum Relative Humidity Sweep Results}

\section{Test Section $T_{w b}$ (static) and Melt Ratio}

\begin{tabular}{|c|c|}
\hline $\begin{array}{l}\mathrm{P}_{0, \mathrm{i}, \mathrm{T}}=44.8 \mathrm{kPa}(6.5 \mathrm{psia}) \text { and } 42.8 \mathrm{kPa}(6.21 \mathrm{psia}) \\
\mathrm{T}_{0, \mathrm{i}, \mathrm{T}}=7.2^{\circ} \mathrm{C} \\
\left.\mathrm{TWC}_{\mathrm{e}, \mathrm{T}}=6.5 \mathrm{~g} / \mathrm{m}^{3} \text { ( }^{*} \text { Estimated }\right)\end{array}$ & \begin{tabular}{lll|} 
& $\mathrm{T}_{\mathrm{wb}, 0, \mathrm{e}, \mathrm{on}, \mathrm{c}}$ & $\mathrm{T}_{\mathrm{wb}, \mathrm{s}, \mathrm{e}, \mathrm{on}, \mathrm{c}}$ \\
& $\mathrm{T}_{\mathrm{wb}, 0, \mathrm{e}, \mathrm{off}} \square$ & $\mathrm{T}_{\mathrm{wb}, \mathrm{s}, \mathrm{e}, \mathrm{off}}$ \\
$\diamond$ & $\eta_{\mathrm{e}}$
\end{tabular} \\
\hline
\end{tabular}

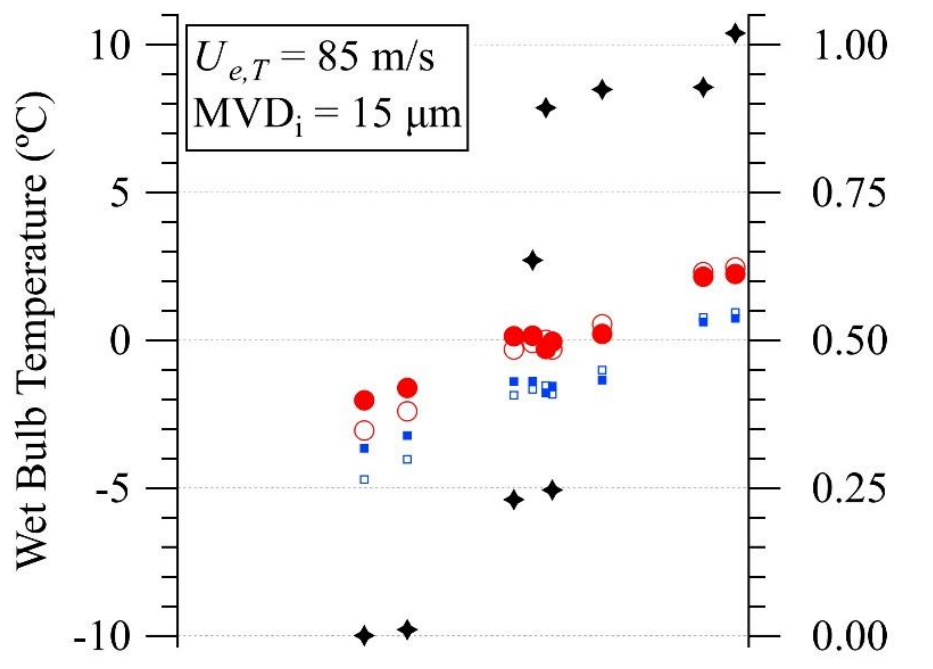

Ice accretion cases $\rightarrow$ a $*$

$\begin{array}{lllllll}0 & 10 & 20 & 30 & 40 & 50 & 60\end{array}$

Relative Humidity Plenum (\%)

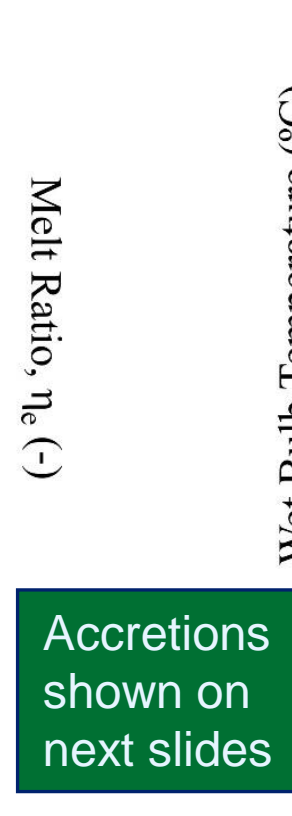

0

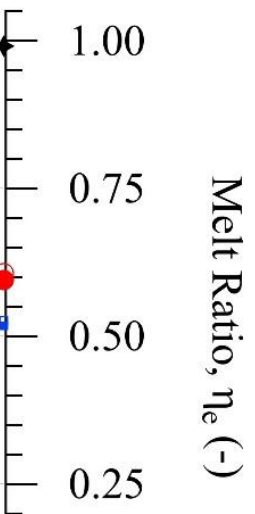




\section{Ice Accretion Examples}

Accretion " $b$ " Low melt ratio 8x actual speed

(10 minute spray time)
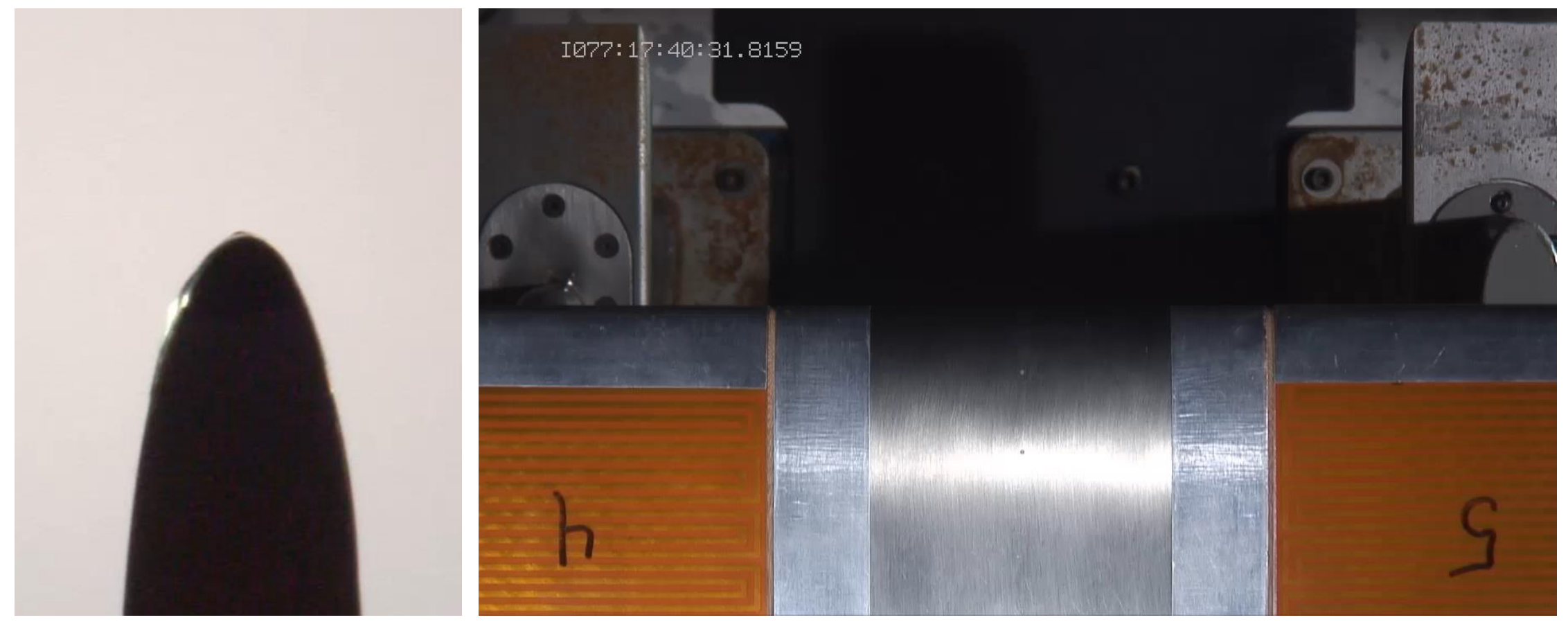


\section{Ice Accretion Examples}

Accretion " $e$ " High melt ratio 8x actual speed (10 minute spray time)
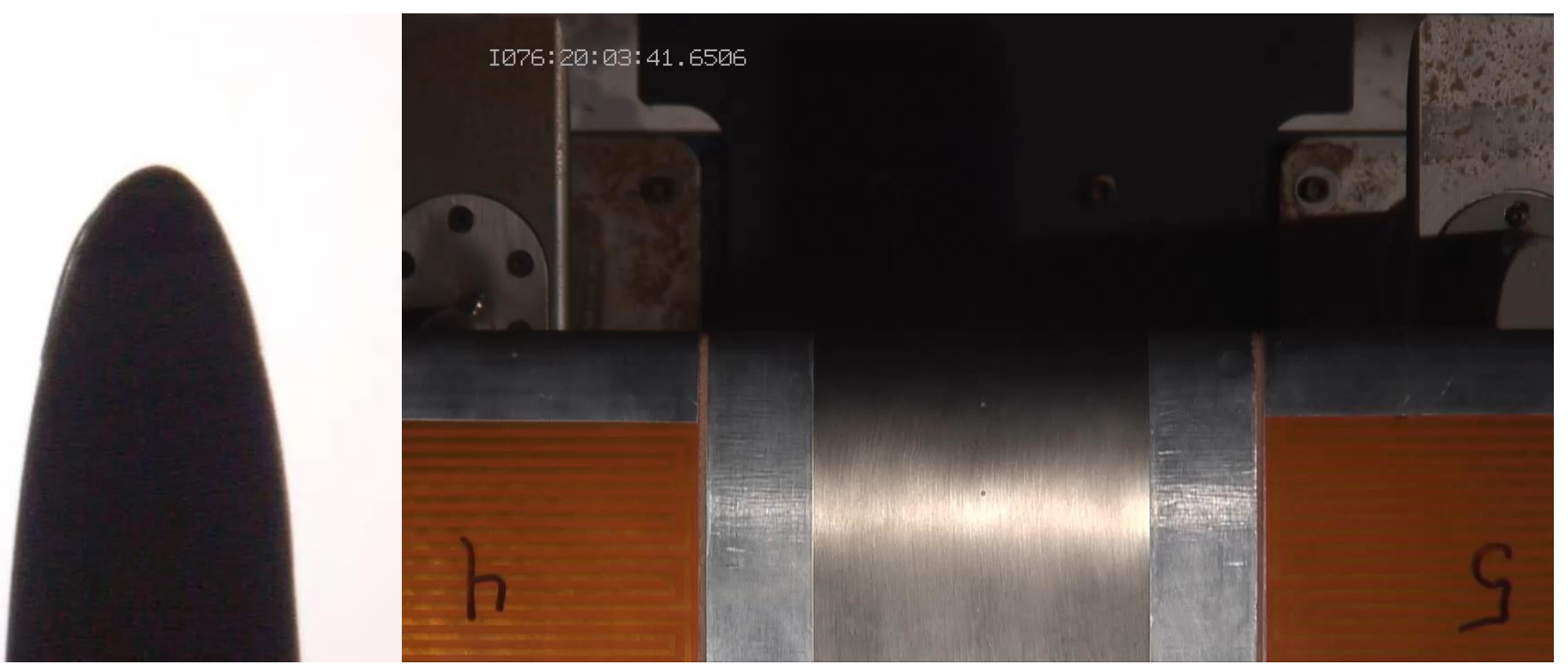


\section{Surface TC meaurements}

\section{Accretion " $b$ "}

\section{Low melt ratio}

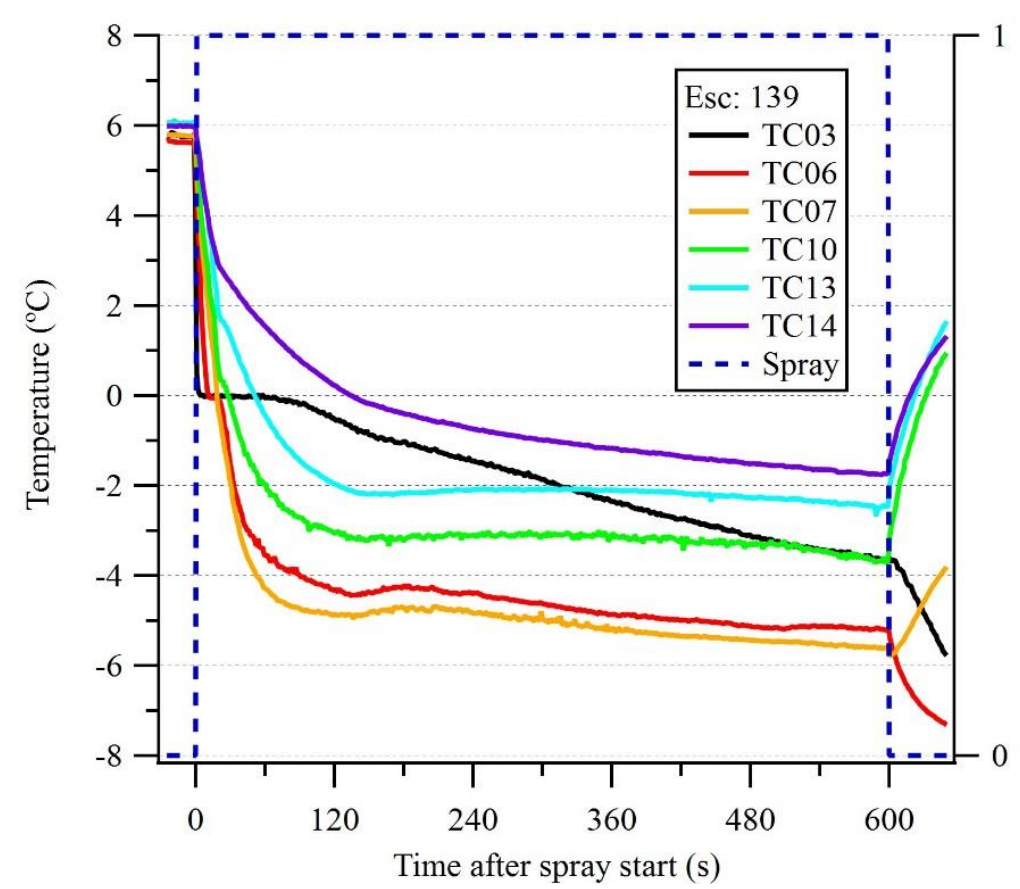

\section{Accretion " $e$ " \\ High melt ratio}

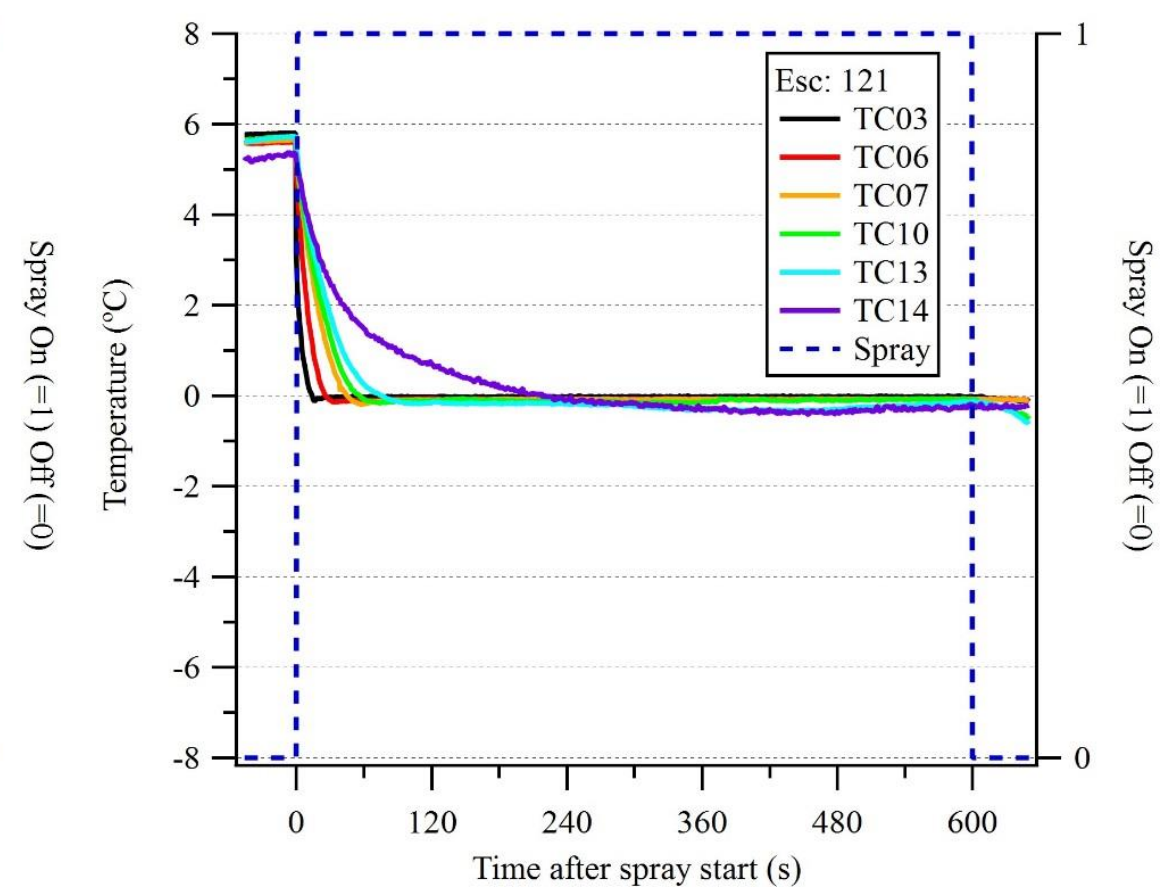




\section{Summary}

- NASA conducting research on fundamentals of $\mathrm{ICl}$ :

- Identify and bound the conditions at the (local) accretion site

- Generate \& characterize conditions

- Develop models \& gather data on ice-crystal icing factors

- Generate environment outside of an engine to facilitate study

- Using PSL as test bed

- Presented data from an 8-day test effort in March 2016, examining:

- Freeze-out characteristics of cloud

- Changes in aero-thermal conditions at the test section

- Ice characteristics observed

- These result offer modelers a dataset to help develop and validate icecrystal, mixed-phase accretion models. 


\section{Acknowledgement}

- Financial support:

- NASA's, Advanced Aircraft lcing (AAI) Subproject

- Mr. Tony Nerone, Project Manager

- Special thanks to:

- Staff of the NASA PSL

- Mr. Chris Lynch for his excellent imaging work. 


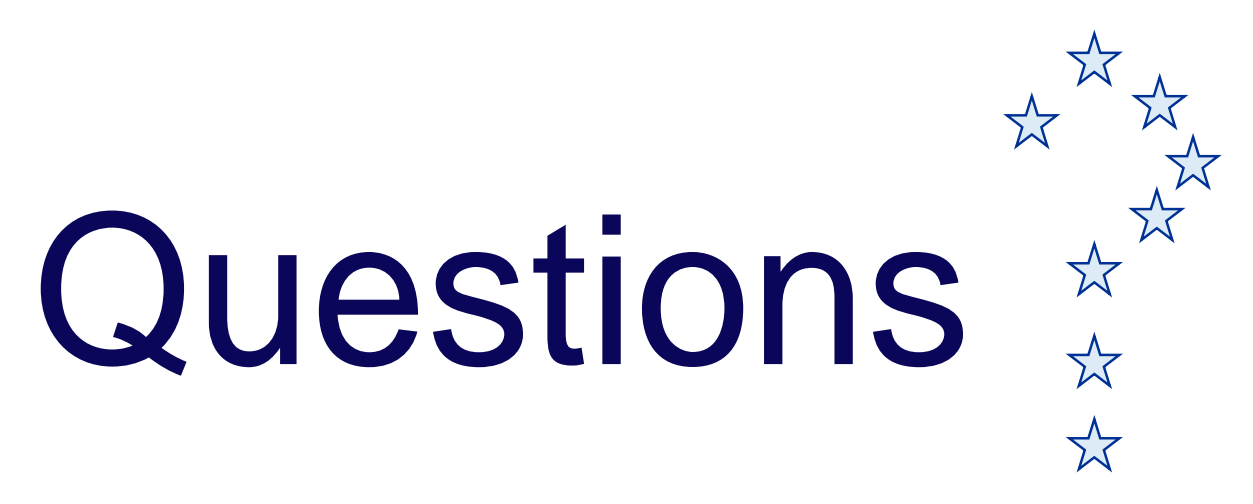




\section{Measurements}

- Temperature

- Rearward Facing Temperature Probe

- Commercial TAT Sensor

- Humidity

- Spectra sensor WVSS-II

- Pressure / velocity / Mach

- Uniformity

- Traverse RTFP

- Temperature

- Humidity

- Condensed phase water

- PSL Tomography
- Total water content

- Isokinetic Probe - version 2

- Liquid water content

- SEA Multi-Element Probe

- Particle size distributions

- Cloud Droplet Probe (CDP)

- Cloud Imaging Probe (CIP)

- High Speed Imager (HSI)

- Phase Doppler Interferometer (PDI)

- Video cameras recorded ice accretion 


\section{Mixed-Phase Investigation}

\section{Plenum Relative Humidity Sweep Results}

\section{Test Section Changes is T and $\omega$ when cloud activiated}

$$
\begin{aligned}
& \mathrm{P}_{0, \mathrm{i}, \mathrm{T}}=44.8 \mathrm{kPa}(6.5 \mathrm{psia}) \text { and } 42.8 \mathrm{kPa}(6.21 \mathrm{psia}) \\
& \mathrm{T}_{0, \mathrm{i}, \mathrm{T}}=7.2^{\circ} \mathrm{C} \\
& \left.\mathrm{TWC}_{\mathrm{e}, \mathrm{T}}=6.5 \mathrm{~g} / \mathrm{m}^{3} \text { ( }^{*} \text { Estimated }\right)
\end{aligned}
$$
